\title{
EXPERIENCIAS DE TERAPIA OCUPACIONAL EN SALUD MENTAL INFANTO JUVENIL: UNA APROXIMACIÓN A PRÁCTICAS DE DERECHOY PARTICIPACIÓN
}

\author{
OCCUPATIONAL THERAPY EXPERIENCES OF CHILD AND YOUTH MENTAL HEALTH: \\ AN APPROACH TO RIGHTS AND PARTICIPATION PRACTICES
}

\section{María José Poblete A. ${ }^{1}$, Francisco Troncoso F. ${ }^{2}$ y Rodrigo Burgos A. ${ }^{3}$}

\section{Resumen}

El presente artículo corresponde a un ensayo que tiene por objetivo mostrar experiencias de intervención de terapia ocupacional en el ámbito de la salud mental infanto juvenil. Se pretende reflexionar en cómo esta disciplina desde su quehacer práctico profesional contribuye a garantizar los derechos, desde las atenciones en dispositivos de salud.

El documento está conformado por cuatro experiencias de Intervención en contextos de salud pública que se vinculan con los principios rectores de la Convención internacional de los Derechos del Niño, los cuales se constituyen en los pilares para el ejercicio de todos los derechos que se establecen en el tratado.

Como parte del análisis de este documento se concluye lo relevante que resulta la generación de políticas públicas atingentes a las necesidades de esta población, ya que de no ser así se reproducen prácticas normalizantes que atentan contra la autonomía de sujetos. En este sentido es fundamental realizar intervenciones situadas que promuevan la inclusión y participación de niños, niñas y jóvenes como actores sociales.

\section{Palabras clave:}

infanto juvenil, derechos, salud mental, terapia ocupacional, participación.

\begin{abstract}
This article corresponds to an essay that has for purpose to show occupational therapy experiences of intervention in child and adolescent mental health. It is intended to reflect how this discipline, from the professional practice, contributes to guarantee rights, from health care devices.

The document has four intervention experiences in public health context, that are bound to the founding principles of the international Convention of Children Rights, which constitute the pillars for the exercise of the rights established in the treaty.

As part of the analysis of this document, it is concluded how relevant it results the development of public politics related to the needs of this population; because if not, "normal practices" are reproduced, and they affect the autonomy of the subject.

In this way, it is important to develop located interventions, to promote the inclusion and participation of boys, girls and young boys as social actors.
\end{abstract}

\section{Keywords:}

child and youth, rights, mental health, occupational therapy, participation.

Fecha de recepción: 30/08/2016.

Fecha de aceptación: 21/10/2016.

1 Terapeuta Ocupacional. Magíster disciplinar con mención en Intervención psicosocial. Terapeuta Ocupacional del Programa Infanto Juvenil, COSAM Macul. Académica Universidad de Chile. Código postal: 7850000. Teléfono: +569 98741561. Email: mjpoblete@med.uchile.cl.

2 Terapeuta Ocupacional. Magíster disciplinar con mención en Intervención psicosocial. Terapeuta Ocupacional del Programa ambulatorio intensivo, COSAM Ñuñoa. Académico Universidad de Las Américas. Código postal: 7520346. Teléfono: +569 85627966. Email: troncoso.fra@gmail.com.

3 Terapeuta Ocupacional. Magíster disciplinar con mención en Intervención psicosocial. Académico Universidad de Las Américas y Universidad Bernardo O’Higgins. Código postal: 8330079. Teléfono: +569 96102614. Email: roburgos@gmail.com. 


\section{INTRODUCCIÓN}

Es sabido que las problemáticas de salud mental son un desafío para la salud pública, las cuales deben ser abordadas en sus distintas dimensiones, contemplando su progresivo incremento en Chile. Bajo este escenario se reconoce que la mitad de los trastornos mentales que sufre la población adulta provienen de la infancia y adolescencia, por ello su presencia genera a lo largo de la vida graves afectaciones al desarrollo emocional, intelectual, familiar y social de quienes los presentan (MINSAL, 2013). Según el último estudio de Carga de Enfermedad y Carga Atribuible (2007) realizado en nuestro país, un $23,2 \%$ de los años de vida perdidos por discapacidad o muerte están determinados por las condiciones neuropsiquiátricas. Para los niños entre 1 y 9 años, un 30,3\% de los Indicadores de Años de Vida Saludable (AVISA) se deben a estas condiciones, proporción que se incrementa a un $38,3 \%$ entre los 10 a 19 años. Es por esto que la propuesta del nuevo Plan Nacional de Salud Mental aboga frente a esta necesidad de la población, ya que se reconocen sus brechas de implementación en donde existe menor oferta de servicios de atención de salud mental para niños, niñas y jóvenes.

Es por esta razón que en la infancia, al igual que en otras etapas, los servicios generados desde el área de la salud deben resguardar el bienestar, previniendo la insatisfacción de las variadas, particulares y cambiantes necesidades de niños y niñas. Paralelamente, en su intervención debe integrar herramientas que garanticen el desarrollo de su autonomía en el ejercicio pleno de sus derechos, en conjunto con la familia, la comunidad y organismos representantes de otras políticas del Estado (Cabieses et al., 2016).

En tanto, con los jóvenes existen una serie de desafíos pendientes en materia de salud. Si bien en los últimos años se ha podido observar un avance en esta materia a través de las acciones y programas llevados a cabo por la institucionalidad en salud y por la sociedad civil, aún existen importantes vacíos producto de los años de invisibilización de las vulnerabilidades de este segmento y sus consecuencias en la salud mental. Esto ha llevado a que en el abordaje de este grupo y prime todavía una visión individual, de conductas de riesgo, que responsabiliza a los/as adolescentes y jóvenes respecto a su salud. Esto ha dado como resultado la invisibilización de las necesidades específicas de salud, sin considerar su opinión en las decisiones que les conciernen (Cabieses et al., 2016).
Resulta necesario e inherente que el Estado garantice la salud mental de niños, niñas y jóvenes, ya que de no dar respuesta a estas problemáticas se conduciría a una condición de vulnerabilidad social, complejizando la situación de salud de los sujetos y vulnerando sus derechos. Debido a lo anterior, el documento muestra prácticas e intervenciones de terapia ocupacional que hacen frente a esta vulneración, considerando de manera primordial el proceso de construcción social y de identidad en diferentes espacios de intervención, donde se expresa cada praxis terapéutica y cómo ésta se adapta a las sensibles realidades individuales, situando a los interventores y equipos de salud mental infanto-juvenil en un constante contexto de búsqueda de soluciones, modalidades y métodos de intervención pertinentes a las problemáticas psicosociales de los sujetos amparados bajo la Convención Internacional de los Derechos del Niño (CIDN).

\section{La salud mental como un derecho de niños, niñas y jóvenes}

Durante la lectura se pretende poner en tensión la realidad actual del ámbito de la salud mental infanto juvenil, las orientaciones y lineamientos desde la política pública en nuestro país referente a este grupo etáreo y cómo, a través de prácticas de terapia ocupacional que se enmarcan bajo el alero de la CIDN, se favorecen procesos de inclusión social.

Es importante señalar que Chile ratificó la Convención Internacional de los Derechos del Niño el 14 de agosto de 1990, el que se rige por cuatro principios fundamentales: la no discriminación, su supervivencia, desarrollo y protección, su participación, así como el interés superior del niño. Este último otorga al/la niño/a el derecho a que se considere y tenga en cuenta de manera primordial su interés superior en todas las medidas o decisiones que le afecten, tanto en el campo de lo público como en lo privado. Estos principios rectores se constituyen en la base para el ejercicio de los derechos de niños, niñas y jóvenes, así como deberían ser las orientaciones éticas en la práctica cotidiana de intervención.

En términos generales, la CIDN establece que los países que se adhieren a estos principios deberán asegurar que todos los niños y niñas se beneficien de una serie de medidas especiales de protección y asistencia; tengan acceso a servicios como la educación y la atención de salud especializada; puedan desarrollar plena- 
mente su personalidad, habilidades y talentos; crezcan en un ambiente de felicidad, amor y comprensión; y reciban información sobre la manera en que pueden alcanzar sus derechos participando en el proceso de una forma accesible y activa (UNICEF, 2006).

En la actualidad se releva la mirada adulto-céntrica sobre los niños, niñas y jóvenes. Esta mirada históricamente los ha posicionado como sujetos carentes y vulnerables, en particular a la hora de compararlos con el mundo adulto. Tal antecedente estructura una oferta de servicios con escaso reconocimiento de las oportunidades y capacidades de éstos/as y sin la incorporación desde una perspectiva de género. Lo anterior impacta en la implementación de intervenciones, las cuales son poco pertinentes a la realidad del mundo infantil y juvenil, considerando el éxito en la intervención desde parámetros e indicadores de logro con un sesgo "adultizado", que no se sitúa en la realidad de esta población, perpetuando en ese quehacer una vulneración (Cabieses et al., 2016).

Entonces, tal como establece el enfoque de derechos propio de la CIDN, "las necesidades de los niños han de ser entendidas como vulneración de derechos y no como carencias, falencias e imposibilidades que impliquen la irreversibilidad de tales situaciones. Cuando son tomadas como vulneración y/o falta de derechos pueden ser perfectamente restablecidas".

La CIDN contiene obligaciones jurídicamente vinculantes sobre el derecho del niño/a al disfrute del más alto nivel posible de salud y a servicios para el tratamiento de las enfermedades y la rehabilitación de la salud (art. 24). No obstante, los avances en lo que respecta a políticas de salud mental aún resultan escasos ya que se han presentado dificultades en la distribución de recursos; a nivel legislativo no se cuenta con una ley de salud mental que garantice el desarrollo, y en particular con la población infanto juvenil los recursos destinados no han sido suficientes, lo que deja en evidencia la necesidad de visualizar, reestructurar y poner en tensión nuestra práctica actual para brindar una atención en salud atingente a las problemáticas reales de este grupo etáreo.

\section{Salud mental infanto juvenil: aportes de la terapia ocupacional}

Para satisfacer las crecientes y diversas necesidades de la población, el Plan Nacional de Salud Mental (1993) postula que se requiere una red de servicios de salud mental y psiquiatría en donde las distintas unidades ofrezcan una amplia gama de prestaciones, se coordinen entre sí para el intercambio de información, la definición dinámica de las respectivas áreas de competencia, el flujo de usuarios/as entre ellas, y la realización de actividades conjuntas, incluyendo tanto la promoción y protección de la salud mental, como el tratamiento y la rehabilitación de las enfermedades, considerando la participación de los/as involucrados/ as y sus familias.

Si bien se pone de manifiesto la necesidad de implementar políticas públicas que otorguen cobertura a quienes presentan problemáticas de salud mental, el posicionamiento ante estas problemáticas requiere de profundas modificaciones para la situación de niños/as y jóvenes, contemplando un abordaje interdisciplinario e intersectorial.

En este sentido, hablar exclusivamente desde la terapia ocupacional en el campo de la salud mental infanto juvenil podría constituirse como un sesgo y una visión desintegrada frente a situaciones y dificultades de sujetos y grupos que merecen una mirada más amplia, intersectorial e inclusiva para poder pretender dar un alcance a la intervención. Lo anterior es razonable considerando las actuales problemáticas en este campo, las que presentan un origen multidimensional, al que en la actualidad se ha intentado dar respuesta desde la medicalización, el control social y la normalización desde figuras de poder y modelos que pretenden la homogeneización de personas, dejando variables psicosociales y sociales como una manera de favorecer un abordaje en salud y no como constituyentes en la etiología de problemas o situaciones que afectan la salud y desempeño ocupacional de niños/as y jóvenes.

La terapia ocupacional desarrolla su quehacer en la vida cotidiana de las personas, como escenario de intervención, liberación y transformación. Es en este lugar donde se pretende apoyar la salud mental y la participación sustantiva a través del compromiso con la ocupación.

Entenderemos la salud mental como el desarrollo o deterioro de las distintas dimensiones de la vida individual en sociedad y que responden a las necesidades del individuo, por tanto como un proceso resultante de las condiciones de vida y que da cuenta de una satisfacción tanto material como simbólica desde el establecimiento de vínculos sanos y solidarios, junto al poder tomar decisiones que afecten sus vidas. Estos factores implican una posibilidad de integración, pertenencia y construcción de proyectos (Custo, 2008). 
En base a la conceptualización mencionada, adquiere relevancia la participación de la terapia ocupacional, tomando como eje central la autonomía, la que podría constituirse como el ejercicio ciudadano y democrático de elegir, controlar y decidir aspectos de la propia existencia, considerando al niño/a o joven como protagonista en su quehacer y su proceso en salud mental; y no subestimar sus reales capacidades y competencias ocupacionales en una sociedad "adultizada" que intenta constituirse desde ese lugar, no permitiendo la incorporación de esta población en su legislación, considerando que son quienes conocen y valoran sus reales necesidades. La terapia ocupacional podría contribuir como intermediario entre éstos y la política pública, al ser un lugar de poder y de implicancias en la salud mental en Chile, a través de evidencia en cuanto al abordaje de prácticas innovadoras y emergentes que responden a los intereses y necesidades de este grupo que se constituye como vulnerable, al no poder dar respuestas a esta condición.

Considerando lo anteriormente expuesto, la terapia ocupacional podría contribuir a prevenir la salud de los niños y niñas a través del compromiso con las ocupaciones que desempeñan, ofreciendo visiones de posibilidades alternativas al contexto de privación ocupacional en el que viven producto de factores y condiciones psicosociales.

A continuación se presentan cuatro experiencias de intervención en salud mental de terapia ocupacional en diferentes dispositivos de atención según complejidad de distintas comunas de la Región Metropolitana ${ }^{4}$, donde participan terapeutas ocupacionales en su rol de interventores, y miembros de un equipo de intervención, promotores de mejorar la calidad de vida de los/as usuarios/as y sus familias, así como el desempeño ocupacional en los diferentes contextos de participación y orientado por la expresión de las dificultades señaladas de forma explícita e implícita. En éstas se muestra el aporte de esta disciplina para el alcance de los principios rectores de los derechos de niñas, niños y jóvenes.

4 En las experiencias a presentar se resguarda la confidencialidad de los antecedentes personales de los sujetos, dando énfasis a los procesos de intervención y los resultados obtenidos a partir de éstos. Tales procesos de intervención se presentan de forma retrospectiva, lo que permite respetar aspectos bioéticos de dichas experiencias.

\section{I.- Interés superior del niño}

Establece que una consideración primordial de los Estados en todas las medidas concernientes a los niños será su "interés superior". Por lo tanto, los Estados deben estar en condiciones de demostrar cómo se ha respetado el principio del interés superior del niño en la adopción de decisiones y cómo se ha sopesado frente a otras consideraciones (SIPI, s.f.).

Acompañamiento terapéutico: Un desafío individual, socio-familiar e institucional. La experiencia a relatar se encuadra en un dispositivo de atención secundaria de salud mental, el cual realiza intervenciones promocionales de carácter intersectorial con un rol protagónico de la comunidad en la creación de entornos que favorezcan el bienestar y la calidad de vida de los individuos y disminuyan los factores de riesgo psicosocial.

Si bien desde este dispositivo correspondiente al sector poniente de la Región Metropolitana no se contemplaba la prestación de acompañamiento terapéuti$\mathrm{Co}$, tras la implementación de esta experiencia el equipo de intervención comienza a dialogar sobre el valor terapéutico de dicha praxis. Realizar acompañamiento terapéutico en el contexto sanitario de nuestro país resulta complejo cuando las prioridades de intervención están enfocadas en acciones costo/efectivas, que no se sitúan desde la realidad de los jóvenes. Bajo este escenario es que se debe comprender que los cambios a los cuales se ven atados, no son sólo desde una perspectiva biologicista, sino que a nivel de la esfera familiar y social. Así lo señala Erikson (2000) en su teoría del desarrollo psicosocial al conceptualizar que los cambios impactan en las relaciones interpersonales, espacio de socialización fundamental en esta etapa, donde los jóvenes en proceso de construcción intentan vincularse con pares formando grupos de interés (influenciado por el ambiente socio-político-geográfico) que aprueben sus nuevas formas de exploración, afianzando su identidad y auto-concepto.

Es por esto que se plantean tres lineamientos de intervención: el primero enfocado en el sujeto, en la construcción de significado que le da a su situación; luego a la reincorporación y participación activa en los diferentes contextos de los cuales participaba y que producto de su situación de salud se margina exponencialmente; y por último un abordaje familiar considerando que el usuario está en dicha situación no solo como individuo, sino también como causal de una estructura familiar igualmente comprometida. 
En este caso la función del acompañamiento terapéutico, como lo señala Kuras de Mauer y Resnizky (2003), es a un nivel vivencial, que presta su persona como referente que permite la regulación en el estado del sujeto, practica y vivencia un rol de protección fundamentalmente de contención permanente y ayuda a desplegar la capacidad creativa del sujeto alentando a la participación en actividades que sean del interés de éste. Asimismo, al interactuar en lo cotidiano se objetivan las variables subjetivas a priori, entonces se entregan posibilidades de acción para el sujeto, aportando una mirada amplia del mundo objetivo. Al co-vivenciar el mundo interno del sujeto, el acompañante terapéutico facilita la reincorporación, el reencuentro en forma paulatina y dosificada con los contextos de participación significativos.

En este escenario se sitúa la experiencia de acompañamiento terapéutico a un joven de 16 años, quien vive junto a su madre y padre, siendo el menor de tres hermanos. A través de diversas entrevistas no estructuradas en su hogar, es posible acceder a información que contextualiza su situación de salud, asociada a las consecuencias provocadas por un acné fulminante que impacta en su identidad e imagen corporal asimismo, se desencadenan manifestaciones como labilidad emocional, anhedonia, irritabilidad, ansiedad a la exposición y apertura a la experiencia, angustia, desánimo y tristeza. No presenta energía e iniciativa, lo que provocó excluirse de diversas ocupaciones significativas, progresivamente su rol de estudiante y el contexto educativo perdieron sentido y se excluye de los espacios de interacción social con grupos de amigos, compañeros de colegios y voluntariados, limitándose tan sólo a contextos virtuales. Además, restringe su participación en espacios familiares, debido a las alteraciones del ciclo sueño/vigilia, lo que impactaba en su interés de relacionarse en dicho contexto.

En primera instancia, los discursos como "no puedo mejorar porque no hay ayuda para lo que me sucede" son recurrentes y denotan la desesperanza y la dificultad en sostener esta situación por más de un año de evolución, sin ninguna mejora sustantiva a nivel de sus ocupaciones. A través de la narrativa de vida se fueron identificando actividades promotoras de bienestar psicosocial, en donde surgieron ocupaciones ligadas a la actividad física como salir a correr, andar en bicicleta y técnicas circenses (acrobacias en telas), que fueron fundamentales para el planteamiento conjunto de objetivos terapéuticos. La intervención se realizó en el espacio cotidiano, situado y reconocido por el usuario, lo que fue fundamental para que se comprometiera con el proceso y aceptara los constantes desafíos. En este sentido, el acompañamiento se centró en salidas en bicicleta en conjunto con el usuario y en la búsqueda de espacios protegidos en la comunidad para realizar consejerías, lo que permitió establecer, entre otras cosas, el vínculo terapéutico.

Entre los resultados obtenidos del proceso de intervención se encuentran:

- El usuario asume mayores responsabilidades a través de la gestión y coordinación con sus familiares el préstamo de bicicletas para enfrentarse a ambientes distintos al del hogar.

- El joven resignifica su experiencia en torno a la enfermedad, a las secuelas (cicatrices), identidad y auto-concepto, mostrando actitud propositiva en el espacio terapéutico.

- El empoderamiento del usuario y la conciencia individual y familiar en el proceso de reparación de la identidad quebrantada por la situación de salud.

- Se evidencian cambios en la interacción familiar, cuyos integrantes manifiestan una convivencia distinta y mejorada al interior del hogar. En relación, a esto, el usuario refiere que "es un avance que mi papá me pregunte cómo estoy".

- La familia asume mayor protagonismo al visualizar que la situación del joven responde a una problemática de salud mental.

- El grupo familiar tensiona los comportamientos propios de la dinámica del hogar que influyen tanto positiva como negativamente en la situación del usuario. Además, fomentan el buen trato y facilitan la exploración autónoma del usuario en los contextos asistidos.

\section{II.- Principio del derecho a la vida, la supervivencia y el desarrollo}

Constituye el derecho intrínseco a la vida, en donde el Estado debe asegurar el crecimiento y desarrollo saludable de niños y niñas. Esto adquiere relevancia, ya que es en esta etapa en donde se forma la base de la salud física y mental, de la seguridad emocional, de la identi- 
dad cultural y personal y del desarrollo de las aptitudes de los niños y niñas (SIPI, s.f.).

Agentes de socialización: Promotores del desarrollo y de la participación ocupacional. La institución en la que se enmarca la siguiente intervención corresponde a un proyecto piloto equivalente al nivel de atención secundaria de salud que atiende a población infanto juvenil con problemáticas de salud mental asociadas a vulneración de derechos, del sector sur de la ciudad de Santiago. Esta instancia tiene como objetivos realizar diagnóstico y tratamiento complementario desde una perspectiva biopsicosocial y sistémica; establecer acciones de coordinación y colaboración intersectorial con los programas pertenecientes a las redes de infancia de la comuna; y articular y apoyar las redes locales, formales e informales, en pro de la salud mental de esta población. Esta instancia pretende validar un modelo de gestión territorial que permita acercar los servicios públicos a los niños, niñas, adolescentes y su familia, de manera de brindar una atención oportuna y eficiente, disminuyendo los factores de riesgos a los que se encuentran expuestos.

La experiencia a presentar es la de un niño de tres años que es derivado al programa debido a que sería expulsado del jardín infantil por problemas conductuales, que se manifiestan en peleas con sus pares y baja capacidad para adaptarse a las normas del establecimiento, según identifican las educadoras.

El motivo de derivación sin duda resulta inquietante para el equipo que lo recibe considerando la edad y el estado de desarrollo en que se encuentra el niño. Al indagar en la historia de vida, se recaban como antecedentes que durante el proceso de gestación la madre consumió drogas y se expuso a situaciones que implicaban riesgo tanto para ella como para el bebé. Una vez ocurrido el parto, tras distintos procedimientos legales el niño queda bajo el cuidado personal de la abuela materna.

Al contactarse con el jardín infantil, su mayor preocupación eran las reacciones violentas de la abuela cuando hacían referencia a su nieto, aludiendo a que ella presentaría consumo de pasta base, además de ser analfabeta. Estos antecedentes, según referían las educadoras, podrían influir en el comportamiento del niño en el contexto educativo. En tanto, la abuela manifestaba temor por perder el cuidado personal y que éste fuese internado en una residencia de menores, lo que había ocurrido anteriormente con otros de sus nietos. En relación al consumo de drogas por parte de la cuidadora, relata que asiste a tratamiento por esta problemá- tica hace más de tres años, logrando la mantención de la abstinencia. Por su parte, el niño tendía al aislamiento entre sus pares y los adultos, lo que estaba afectando la adquisición de destrezas psicomotoras y socioemocionales, y la capacidad para involucrarse en el juego.

Ante este escenario, resulta relevante en primera instancia identificar los actores sociales con los que se vinculaba el niño, ya que las interacciones sociales establecidas se constituyen en procesos de socialización, en donde se adquieren aprendizajes a lo largo de la vida que nos permiten percibir, distinguir, sentir, organizar y operar en la realidad. De esta manera, a través de los procesos de socialización se forman actitudes se desarrollan competencias para sentirse parte de la sociedad y se comparten creencias, valores, normas de conducta, costumbres, lenguaje y formas de relación con la comunidad. Por lo tanto, el equipo de intervención buscó articular las distintas redes con las que el grupo familiar estaba conectado con el fin de desmitificar y clarificar la situación del niño, generando instancias de comunicación que permitieran el acceso a distintos servicios que favorecen su desarrollo. De esta forma el niño mantuvo su asistencia al jardín infantil y junto al grupo familiar recibieron atenciones de salud acordes a sus necesidades.

La articulación con las redes constituye, tal como lo explican Pino y Ceballos (2015), una estrategia de intervención: Pasa por una intervención en la red del sujetofoco con el propósito de desarrollarla operativamente en función de los problemas y necesidades de éste, de reconstruirla o sustituirla en caso de disfuncionamiento y de construirla cuando no existe.

Simultáneamente, los objetivos de intervención de terapia ocupacional con la adulta responsable buscaban desarrollar motivación y confianza percibida por el bienestar personal y el de su nieto con un acompañamiento en el quehacer cotidiano. Con el niño, el abordaje pretendía crear distintas formas ocupacionales que pudiesen aumentar su curiosidad, mantener su atención y facilitar la expresión emocional y las interacciones sociales.

Tras el proceso de intervención del equipo interdisciplinario, y en particular de terapia ocupacional, se evidencian los siguientes resultados:

- La abuela percibe que valoran sus esfuerzos por la protección y cuidados del niño, desarrollando la confianza personal para desenvolverse en distintos ámbitos de su vida cotidiana, incluyendo la posibilidad de recibir apoyo para adquirir la lecto-escritura. 
- La cuidadora problematiza la condición de sujeto de derecho del niño y disminuye la ansiedad generada por la incertidumbre de perder el cuidado personal, identificando cuáles son las necesidades que debe cubrir en el proceso de crianza.

- La adulta incorpora estrategias que permiten manejar las reacciones violentas ya que éstas habrían influido en el comportamiento del niño en el jardín infantil. En este sentido, se facilitó la participación del niño y de su abuela en actividades lúdicas que incorporaron como parte de su cotidianidad.

- En tanto con el niño, si bien muchas de sus conductas respondían al manejo de los adultos que se configuraban en los agentes de socialización, de igual forma fue adquiriendo algunas destrezas psicomotoras acordes a la etapa en la que se encontraba, así como también desarrolló habilidades para relacionarse con sus pares a través del juego. Lo anterior promovió la autonomía e independencia en actividades cotidianas, en función de la evolución de sus facultades, haciéndolo sentirse parte de los contextos en los que se desenvolvía.

\section{III.- Principio de No discriminación}

Establece que no deben realizarse distinciones entre niñas, niños y jóvenes por raza, religión, situación de salud, opinión o habilidades, entre otros. Este principio refleja uno de los pilares fundamentales de los tratados internacionales de derechos humanos.

Taller de habilidades sociales: Desarrollo del sentido de comunidad en la infancia. La práctica a describir emerge de la misma instancia piloto de la experiencia anterior pero de otra zona de la región (sector poniente).

El programa contaba con lineamientos generales de funcionamiento, debiendo situarse en la realidad local. Cabe destacar que resultaba contradictorio porque si bien los paradigmas imperantes en salud mental y psiquiatría promueven un enfoque comunitario, todavía se despliegan estrategias que responden a un posicionamiento biomédico. Pero aún así se busca alcanzar una práctica comunitaria promotora de la salud mental y del bienestar psicosocial. Para situarnos en la práctica comunitaria es necesario revisar la noción de comunidad. En el mundo natural, nuestra comunidad es la unidad que organiza los movimientos individuales en el espacio a través del tiempo. En el mundo cultural, la comunidad es el marco en el que, de generación en generación, los seres humanos aprenden a desarrollar todo su potencial humano. La comunidad está relacionada con conectividad y es una unidad con profundidad histórica en el tiempo (Kronenberg et al., 2007). En este sentido, el objetivo de las prácticas comunitarias incluyen la resolución de problemas y el desarrollo psicosocial, lo que permite fomentar el poder que las personas pueden ejercer sobre su entorno logrando cambios en esos ambientes y en la estructura social (Montero, 2004). Ese poder transformador es denominado empoderamiento.

Dentro de este marco se comienzan a idear y explorar metodologías de trabajo para implementar este programa. Como estrategia se realizó una alianza de trabajo con establecimientos educacionales. En este caso se hará referencia a la experiencia con una escuela particular subvencionada, desde donde derivaba un alto porcentaje de niños y niñas, quienes recibían atenciones a nivel individual, familiar y social, manteniendo reuniones de seguimiento semanales con la psicóloga y psicopedagoga del colegio.

Los niños y jóvenes que el equipo atendía presentaban diferentes tipos de problemáticas tales como cuadros emocionales, dificultades en sus relaciones interpersonales, problemas conductuales, situaciones de bullying, presencia de maltrato y/o abuso y antecedentes de violencia intrafamiliar, entre muchos otros, que mostraban la complejidad multidimensional de los sujetos de intervención.

Si consideramos que la escuela es un espacio de socialización, estamos teniendo en cuenta que se reproducen patrones de comportamiento y dinámicas sociales propias del contexto. Por ende, es un área que debe ser abordada cuando trabajamos con niños y niñas que son parte de este sistema. Desde un tiempo a otro comenzaron a llegar varios niños de edades que fluctuaban entre 6 a 9 años con dificultades para relacionarse con sus pares afectando la convivencia escolar de sus respectivos cursos, lo que preocupaba tanto a apoderados como a profesores. Cada uno de ellos con sus historias personales y contextos también particulares. Es por esto que con el equipo de intervención se planteó la idea de talleres tanto para los padres como para los niños que permitieran abordar dichas temáticas.

La psicóloga y la terapeuta ocupacional del programa se hicieron cargo del taller de niños, al cual le dieron el nombre de "habilidades sociales", que no se vinculaba necesariamente con lo que se deseaba alcanzar como meta terapéutica, pero institucionalmente era más fácil de entender. Esta intervención tenía como propósito el generar sentido de comunidad en los ni- 
ños, el cual es comprendido como el sentimiento de formar parte de algo mayor, un sentimiento compartido de que las necesidades colectivas serán asumidas con la cooperación de todos (McMillan y Chavis, 1986). A los niños se les mostraron los objetivos como base de esta instancia, el respeto hacia la diversidad, el buen trato entre personas, el conocer sus derechos, el poder escuchar al otro y asimismo expresar las ideas y emociones dentro de un ambiente de confianza.

La planificación del taller contempló sesiones diagnósticas participativas donde se fomentó el vínculo de grupo y se rescataron las opiniones e intereses de los niños. En base a éstos se programaron diez sesiones, las cuales contemplaban también un encuentro con los padres de los niños al finalizar el taller.

El taller se desarrollaba en aproximadamente una hora y media de trabajo semanal, donde gradualmente se fue dando el espacio para poder compartir temáticas de mayor envergadura, siempre en un contexto dinámico y atractivo para ellos. Se utilizaron estrategias de juego terapéutico, dinámicas de grupo, de educación popular, como también estrategias individuales para algunos niños dentro del contexto grupal.

El proceso de intervención grupal alcanzó los siguientes logros:

- Los niños valoran este espacio por las oportunidades de expresarse, trabajar colaborativamente y el respeto que se promovía dentro de éste, proyectándolo a la necesidad de incorporar estas formas de interacción en determinados contextos, entre ellos el escolar.

- Los niños adquieren protagonismo y autonomía para la resolución de problemas y para la generación de espacios de participación; y además reflexionan sobre diversas problemáticas que identifican como relevantes.

- $\quad$ Este espacio de intervención se constituye para los usuarios en la instancia de participación que permite generar lazos sociales y cohesión intragrupal.

- Desde el colegio valoran la instancia y las metodologías utilizadas, visualizando la convivencia escolar como base para el desempeño en este contexto y la necesidad de generar espacios que promuevan la solidaridad recíproca y el respeto mutuo.

\section{IV.- Principio de participación}

Este principio pone de relieve la función del niño como participante activo en la promoción, protección y vigilancia de sus derechos. Establece el derecho de todo niño a expresar su opinión libremente en todos los asuntos que le afecten, y en consecuencia establece que esas opiniones se tengan debidamente en cuenta en función de la edad y madurez del niño (SIPI, s.f.).

Taller de salidas: Una apertura a la participación social, la ocupación y la inclusión social. El siguiente espacio grupal emerge bajo la identificación y cotejo de necesidades, expectativas e intereses de un grupo de jóvenes pertenecientes a un programa ambulatorio intensivo para el tratamiento en adicciones del sector oriente de la región, el que tiene por objetivo "Generar una posibilidad de acercamiento a la comunidad de forma protegida, participativa e inclusiva, que favorezca la participación social, la autonomía y la accesibilidad a espacios culturales, territoriales, artísticos, entre otros, como sujetos sociales activos". Para llevar a cabo lo anterior se utilizó una opción teórica, que fundamenta el actuar desde una perspectiva de derecho, inclusiva, así como la utilización de la vida cotidiana como el escenario de intervención y transformación de la realidad. Y entendida como el lugar donde se lleva a cabo la construcción de los sujetos, mediante prácticas sociales que se cristalizan en las relaciones intersubjetivas, discursos, objetos significados, entre otros.

Desde esta premisa entenderemos entonces la vida cotidiana como un escenario legítimo de intervención, donde la reproducción naturalizada e irreflexiva de ésta cristalizará prácticas sociales de acuerdo a la historicidad, el momento social actual y los lineamientos macro-sociales en los cuales nos encontramos inmersos. En relación a esto se desprende el carácter biográfico que arrastra cada uno de los sujetos a intervenir, en donde la cotidianidad adquiere un papel de relevancia. Heller (1972) y plantea que la vida cotidiana es el espejo de la historia, también la define "como el conjunto de actividades que caracterizan la reproducción de los hombres particulares, los cuales crean la posibilidad de la reproducción social... es la forma real en que se viven los valores, creencias, aspiraciones y necesidades".

Para Pichón-Rivière es coherente al sostener que: "entiendo al hombre como configurándose en una actividad transformadora, en una relación dialéctica, mutuamente modificante con el mundo, relación ésta que tiene su motor en la necesidad" (Pampliega de Quiroga, Racedo, 1993). 
Toda práctica de terapia ocupacional se realiza en la vida cotidiana, por lo que debe realizarse bajo un proceso reflexivo por parte de los interventores e intervenidos. De esta manera se generan prácticas que contribuirán a la transformación social y en generar reales cambios que favorezcan la inclusión. Desde esta lógica es posible pensar en la vida cotidiana como el espacio de reflexión y problematización de los procesos interventivos y perfilarse como la estrategia de liberación de la enajenación y control social que se le adjudica a la condición actual de estos usuarios/as.

En este contexto y en relación a elementos implícitos que se encuentran al interior de participación social, tales como "ciudadanía", "accesibilidad", "derecho", entre otros. También existen aspectos de igual importancia que no son tan visibles. Liliana Paganizzi (2007) explícita que inherentes a la participación y autonomía se encuentran elementos relativos a la posibilidad de decidir y controlar aspectos que cruzan la vida de los sujetos; es por lo anterior que este espacio grupal tuvo una metodología participativa y co-construida. La autora refiere que elementos tales como "la forma de comer, vestirse, higienizarse, manejar dinero y trasladarse soportan funciones subjetivas y sociales cargadas de connotaciones simbólicas que se tornan indispensables para la coexistencia comunitaria y la participación social" (Paganizzi, 2007). Elementos centrales que serán parte de la intervención en espacios de exploración ocupacional y objetivos a trabajar en la vida cotidiana.

En términos metodológicos se propusieron los siguientes objetivos de trabajo, los cuales dieron respuesta de manera satisfactoria para los/as usuarios/as y el equipo de intervención en las experiencias grupales de tratamiento en adicciones con juventudes. Como objetivo general se planteó "Que los/as jóvenes del programa logren generar una posibilidad de acercamiento a la comunidad de forma protegida, participativa e inclusiva, que favorezca la participación social, la autonomía y la accesibilidad a espacios culturales, territoriales, artísticos, etc. como sujetos sociales activos".

De manera más específica se logró que las y los jóvenes:

- Participaran en la organización y planificación de las diferentes salidas acordadas de manera conjunta.

- Desarrollaran habilidades de organización y planificación y cumplimiento de acuerdos acorde a sus propias expectativas al interior del espacio grupal
- Adoptaran e incorporaran en su desempeño cotidiano habilidades sociales de base y competencias ocupacionales.

- Desarrollaran habilidades de la vida diaria instrumental ligadas al uso de transporte público y manejo de dinero, y que participaran en espacios públicos y privados acorde a las características de cada lugar.

- Redujeran sus tiempos libres y de ocio destinados en mayor medida a la actividad delictual y de consumo de sustancias psicoactivas.

- Ampliaran su red social focal en el cuadrante comunidad, con espacios de participación sostiene de carácter prosocial.

- Visualizaran la comunidad como un espacio del cual ellos/as también son parte y sujetos de ella.

- Conocieran e identificaran espacios recreativos, sociales, culturales y artísticos de su interés, desde una perspectiva responsable e inclusiva.

\section{COnCLUSIONES}

Luego de este ejercicio disciplinar parece razonable poder detenernos en lo que respecta a las diferentes expresiones de infancias y juventudes. En este contexto se torna necesario poder dar más atención y considerar las distinciones entre el variopinto escenario de estas diferentes poblaciones. Considerando lo anterior, resulta fundamental pensar en la creación de políticas públicas y orientaciones técnicas que respondan a las reales necesidades y de forma específica a estas comunidades históricamente vulneradas y marginadas de espacios de opinión como ejercicio de derecho. Solo de esta manera no estaríamos incurriendo en una reproducción de prácticas normalizantes y adultocéntricas, sin dar espacio a la validación de la diferencia y por qué no pensar en vulneraciones de derechos al no dar respuesta a las necesidades específicas de determinadas poblaciones.

La terapia ocupacional y los/as terapeutas ocupacionales se sitúan en un lugar protagónico, en cuanto ejecutores de política pública, reproduciendo o facilitando la transformación de la realidad de niños/as y jóvenes. Es en este ámbito que toma relevancia el ejercicio político y social de develar pequeños espacios de libera- 
ción y prácticas emancipadoras como las presentadas en este artículo, en donde en un esfuerzo profesional, valórico y de derechos se ha podido dar respuesta a necesidades de sujetos y colectivos, la que en oportunidades implica ir en contra de lo institucional, la orientación técnica o la política pública que se expresa en las diferentes prácticas disciplinares. Asimismo, tensionar el rol que ejerce el sistema de salud, donde el foco de atención no está al nivel de la promoción y prevención de salud mental, sino más bien al nivel del tratamiento y rehabilitación; por ende, hay que cuestionar esta prioridad del sistema que pone énfasis en la enfermedad y la psicopatologización de conductas inherentes de niños, niñas y jóvenes, perdiendo la capacidad de observar de manera global, contextual y situada el desarrollo psicosocial de estos sujetos. En este sentido, consideramos que la disciplina debe asumir una posición que genere problematización conjunta con el entramado social que sostiene a este sujeto en construcción, fomentando la co-responsabilidad que tienen los garantes de derechos en la construcción de subjetividades de los/as niños/as y jóvenes.

Nos parece responsable, desde un deber ético, poder expresar estas líneas en pro de compartir y socializar experiencias de intervención reales y efectivas tanto en niños y niñas como en jóvenes y grupos excluidos de nuestro país, considerando la escasa red de saber y de verdad frente a la intervención y los procesos en estos ciclos vitales. Es sabido lo escaso que es la producción de conocimiento en el área de salud mental infanto juvenil, sin embargo este precedente no debería ser naturalizado por aquellos/as que nos encontramos en este campo de intervención, muy por el contrario, consideramos que los esfuerzos deberían doblegarse para aportar en la construcción del conocimiento idóneo y especializado que merecen los/as usuarios/as en nuestro quehacer.

Para finalizar, resulta necesario observar nuestra praxis y revisar aquellos sustentos teóricos con los cuales operamos como profesionales, tensionar los posicionamientos paradigmáticos con el objetivo de desarrollar una práctica coherente y centrada en las necesidades del sujeto de intervención, tensionar marcos de referencia y modelos de intervención propios en nuestro quehacer disciplinar, incluir en éstos transformaciones que involucren elementos culturales, sociales y políticos, que no podemos dejar atrás debido a que avanzan de forma súbita e irreflexiva, incorporar los estilos de vida y las cotidianeidades de los diferentes grupos sociales y en particular de los/as niños/as y jóvenes que han ex- perimentado cambios a lo largo de nuestra historia De esta manera, consideramos que se podrá avanzar hacia una mirada en donde no recaiga toda la responsabilidad en el sujeto, sino que en un entramado social y político que también debiese replantearse para el beneficio de ciudadanos/as en igualdad de condiciones.

\section{AgradeCIMIENTOS}

A todos y cada uno de los/as usuarios/as y sus familias que el destino y las circunstancias sociales han permitido que nos encontremos y aprendamos los unos de los otros.

A la terapeuta ocupacional María Ignacia Navarrete, quien desde su experiencia busca contribuir al desarrollo disciplinar a favor de los usuarios/as con los que trabaja.

\section{REFERENCIAS BIBLIOGRÁFICAS}

Cabieses, B., Bernales, M., Obach, A. Pedrero, V. (2016). Vulnerabilidad social y su efecto en salud en Chile: Desde la comprensión del fenómeno hacia la implementación de soluciones. Santiago: Universidad del Desarrollo, Equipo de Estudios Sociales en Salud.

Chile, Ministerio de Salud (2013). Programa Nacional de Salud de la infancia con enfoque integral. Santiago: Ministerio de Salud

Chile, Ministerio de Salud (1993). Políticas y Plan Nacional de Salud Mental. Santiago: Ministerio de Salud.

Chile, Ministerio de Salud (2007). Segundo Estudio de Carga de Enfermedad y Carga Atribuible. Santiago: Departamento de Epidemiología. Universidad Católica de Chile.

Custo, E. (2008). Salud Mental y Ciudadanía. Una perspectiva desde el Trabajo social. Buenos Aires: Espacio Editorial.

Erikson, E. (1985). El ciclo vital completado. Buenos Aires: Ed. Paidós.

España, UNICEF, (2006). Convención sobre los Derechos del Niño. Madrid: UNICEF.

Heller, A. (1972). Historia y vida cotidiana: aportación a la sociología socialista. México: Ed. Grijalbo.

Kronenberg, F., Simó Algado, S. y Pollard, N. (2007). Terapia Ocupacional sin fronteras: aprendiendo del espíritu de supervivientes. Madrid: Ed. Panamericana.

Kuras de Mauer, S. y Resnizky, S. (2013). Acompañantes Terapéuticos: Actualización Teórico - Clínica. Ed. Letra Viva: Argentina.

McMillan, B. \& Chavis, D. M. (1986). Sense of community: a definition and theory. Journal of Community Psychology, 14, 6-23.

Montero, M. (2004). Introducción a la Psicología Comunitaria, DesarroIlo, conceptos y procesos. Argentina: Ed. Paidós. 
Navarrete, E., Cantero, P., Guajardo, A., Sepúlveda, R., y Moruno, P. (2015). Terapia Ocupacional y Exclusión social: Hacia una praxis basada en los derechos humanos. Lo social y lo comunitario de la Terapia Ocupacional (1a. ed., pp. 29-44). Santiago: Ed. Segismundo.

Oyarzún, N., Zolezzi, R., Núñez, J. y Palacios, M. (2009). Hacia la construcción de las prácticas comunitarias de terapeutas ocupacionales en Chile, desde una mirada socio histórica, desde 1972 hasta la actualidad. Revista Chilena de Terapia Ocupacional, 9, 149-165.

Paganizzi, L. (2007). Terapia ocupacional psicosocial. Escenarios clínicos y comunitarios. Buenos Aires: Ed. Polemos.

Pampliega de Quiroga, A. y Racedo, J. (1993). Crítica de la vida cotidiana. Buenos Aires: Ed. Cinco.

Pavez, I. (2012). Sociología de la Infancia: las niñas y los niños como actores sociales. Revista de Sociología, 27, 81-102.

Pino, J. y Ceballos, M. (2015). Terapia Ocupacional Comunitaria y Rehabilitación Basada en la Comunidad: hacia una inclusión socio comunitaria. Revista Chilena de Terapia Ocupacional, 15(2), 1-15.

Polonio, B., Castellanos, M. y Viana, I. (2008) Terapia Ocupacional en la infancia. Teoría y práctica. Buenos Aires: Editorial Médica Panamericana.

Sistema de información sobre la primera infancia en América Latina SIPI (s.f.). Itinerarios. Los principios generales de la Convención internacional de los Derechos del niño en las leyes y códigos de la infancia. UNICEF. 